\title{
ALTERAÇÕES COMPORTAMENTAIS GRAVES EM PACIENTE COM DOENÇA DE FAHR
}

\author{
Arthur Kümmer1, Maila de Castro' ${ }^{1}$, Paulo Caramelli², \\ Francisco Cardoso ${ }^{3}$, Antônio Lúcio Teixeira ${ }^{4}$
}

\begin{abstract}
RESUMO - Relatamos o caso de um homem de 40 anos de idade com doença de Fahr, definida por calcificações idiopáticas bilaterais em núcleos da base, que desenvolveu transtorno depressivo, tiques motores e fônicos, comportamentos estereotipados tipo "punding" e alterações de personalidade com importantes implicações sócio-familiares. Discutimos as manifestações psicopatológicas da doença de Fahr e a importância dos núcleos da base na determinação do comportamento humano.
\end{abstract}

PALAVRAS-CHAVE: doença de Fahr, núcleos da base, depressão, tiques, punding.

\begin{abstract}
Severe behavioral changes in a patient with Fahr's disease
ABSTRACT - We report on a case of a 40 year-old man with Fahr's disease, defined by idiopathic bilateral basal ganglia calcification, who developed depressive disorder, motor and phonic tics, stereotyped behaviors such as punding and personality changes with significant social and familiar implications. We discuss about the psychopathology of Fahr's disease and the relevance of the basal ganglia in the determination of human's behavior.
\end{abstract}

KEY WORDS: Fahr's disease, basal ganglia, depression, tics, punding.

A doença de Fahr (DF), epônimo para calcinose estriado-pálido-denteada idiopática, refere-se à calcificação idiopática bilateral nos núcleos da base, esporádica ou familiar, supostamente uma doença autossômica dominante ${ }^{1}$. Apesar de ser nomeada em homenagem a Theodor Fahr, a doença é conhecida desde 1850, muitos anos antes da descrição por este em $1930^{2}$. Na realidade, Theodor Fahr relatou o caso de um homem de 81 anos com crises epilépticas e calcificação difusa de vasos cerebrais e núcleos da base relacionada provavelmente a hipoparatireoidismo, não sendo, portanto, idiopática ${ }^{3}$. Ressalta-se que a calcificação nos núcleos da base já foi relacionada a mais de 30 condições clínicas, incluindo uma variedade de doenças infecciosas, metabólicas e genéticas $^{1,4}$. Alguns autores empregam o termo síndrome de Fahr para descrever a calcificação patológica nos núcleos da base que pode ser causada por doenças de etiologias diversas ${ }^{3}$. Entretanto, nem todos os casos de calcificação intracraniana são síndrome de Fahr já que podem acometer outras áreas que não os núcleos da base. De nota, calcificações dos núcleos da base também podem ser encontradas incidentalmente em 0,7 a 1,2\% dos exames de tomografia computadorizada de crânio (TC). Essas calcificações são usualmente benignas, sem etiologia definida e com localização preferencial nos globos pálidos, especialmente em pacientes acima de 60 anos de idade ${ }^{1}$. O termo doença de Fahr é reservado aos quadros idiopáticos de calcificação patológica nos núcleos da base ${ }^{3}$. Os núcleos da base têm sido exaustivamente estudados por estarem relacionados não apenas com a motricidade, mas com diferentes aspectos do comportamento humano, encontrando-se alterações nessas estruturas em transtornos do humor, de personalidade, psicoses e transtornos do chamado espectro obsessivo-compulsivo.

O caso que relatamos diz respeito a um adulto

\footnotetext{
${ }^{1}$ Residente de Psiquiatria do Instituto Raul Soares, Fundação Hospitalar do Estado de Minas Gerais (Fhemig), Belo Horizonte MG, Brasil; '²Médico Neurologista. Professor do Curso de Pós-Graduação em Neurologia da Faculdade de Medicina da Universidade de São Paulo; Professor Adjunto do Departamento de Clínica Médica da Faculdade de Medicina da Universidade Federal de Minas Gerais, Belo Horizonte MG, Brasil (FM/UFMG); ${ }^{3}$ Médico Neurologista, Coordenador da Clínica de Distúrbios do Movimento do Hospital das Clínicas da UFMG, Professor Adjunto de Neurologia da FM / UFMG; ${ }^{4}$ Médico Neurologista e Psiquiatra, Professor Adjunto de Neurologia da FM / UFMG.
}

Recebido 20 Outubro 2005, recebido na forma final 20 Janeiro 2006. Aceito 1 Abril 2006.

Dr. Antônio Lúcio Teixeira Jr - Departamento de Clínica Médica, Faculdade de Medicina / UFMG - Av. Prof. Alfredo Balena 190 30130-100 Belo Horizonte MG - Brasil. E-mail: altexr@gmail.com 
com calcificações idiopáticas em núcleos da base e em outras regiões encefálicas e que desenvolvem transtorno depressivo crônico, tiques motores e fônicos persistentes, além de alterações de personalidade e comportamentos estereotipados e repetitivos denominados "punding". Este tipo de comportamento foi descrito originalmente em indivíduos que faziam uso excessivo de drogas com ação dopaminérgica, como anfetaminas, cocaína ou levodopa, e que teoricamente sensibilizariam o circuito fronto-estriatal ventromedial que exerce importante papel nos fenômenos de recompensa ${ }^{5}$. Salientamos que o "punding" pode ocorrer em outras condições clínicas que interf e rem nos núcleos da base e, conseqüentemente, nos circuitos fronto-estriatais.

\section{CASO}

Homem de 40 anos com $1^{\circ}$ grau completo, representante comercial, evangélico. Pro c u rou nosso serviço queixando-se de "depressão" que caracterizava como tristeza constante, choro ocasional, pessimismo e apatia, sintomas de intensidade leve a moderada, mas que se arrastavam por cerca de 3 anos. A esposa relatava que ele vinha ficando progressivamente mais descuidado com a aparência pessoal e com "hábito de mentira e de comprar tudo". Mostravase agressivo quando confrontado, embora reconhecesse que contava mentiras para a esposa, bem como adquiria dívidas no nome dela por causa de suas compras desnecessárias. Não sabia o motivo por se comportar dessa forma dizendo fazê-lo "sem pensar, de forma impulsiva". Negava sintomas que indicassem episódio hipomaníaco eufórico ou disfórico, nem sintomas obsessivos. A pontuação no Mini-Exame do Estado Mental era 30/30.

De nota na história pre g res sa, exibira epilepsia na infância, tendo usado fenobarbital $100 \mathrm{mg}$ dos 5 aos 15 anos, quando abandonou o tratamento. Desde então, não apresentara mais crises epilépticas. Negava história familiar de transtorno mental e dizia que sua mãe falecera com doença de Parkinson (DP). Foi diagnosticado com distimia, sendo iniciado fluoxetina $20 \mathrm{mg} / \mathrm{dia}$. Um mês depois, apresentou crise epiléptica tônico-clônica generalizada. No período pós-ictal, mostrou-se disártrico, atáxico e com bradicinesia e instabilidade postural. Foi submetido à TC que evidenciou imagens hiperdensas nos núcleos lentiform es e caudado, substância branca frontal profunda, substância branca temporal subcortical (A) e centros semi-ovais (B) (Fig 1). Realizou extensa propedêutica laboratorial para investigar calcificações intracranianas patológicas, incluindo sorologia para hepatites virais, sífilis e HIV, provas de atividade inflamatória, de função renal e hepática, ionograma, TSH, T4 livre e paratormônio, com resultados sem alterações. $O$ eletrencefalograma mostrou-se normal. Recebeu o diagnóstico, então, de DF (calcificação idiopática nos núcleos da base), sendo associado fenobarbital $100 \mathrm{mg} / \mathrm{dia}$. O paciente evoluiu com melhora espontânea dos sintomas cere b e $l$ a res $e$ parkinsonianos até a remissão completa em 4 semanas.

Nos meses seguintes, evoluiu com acentuação prog res-

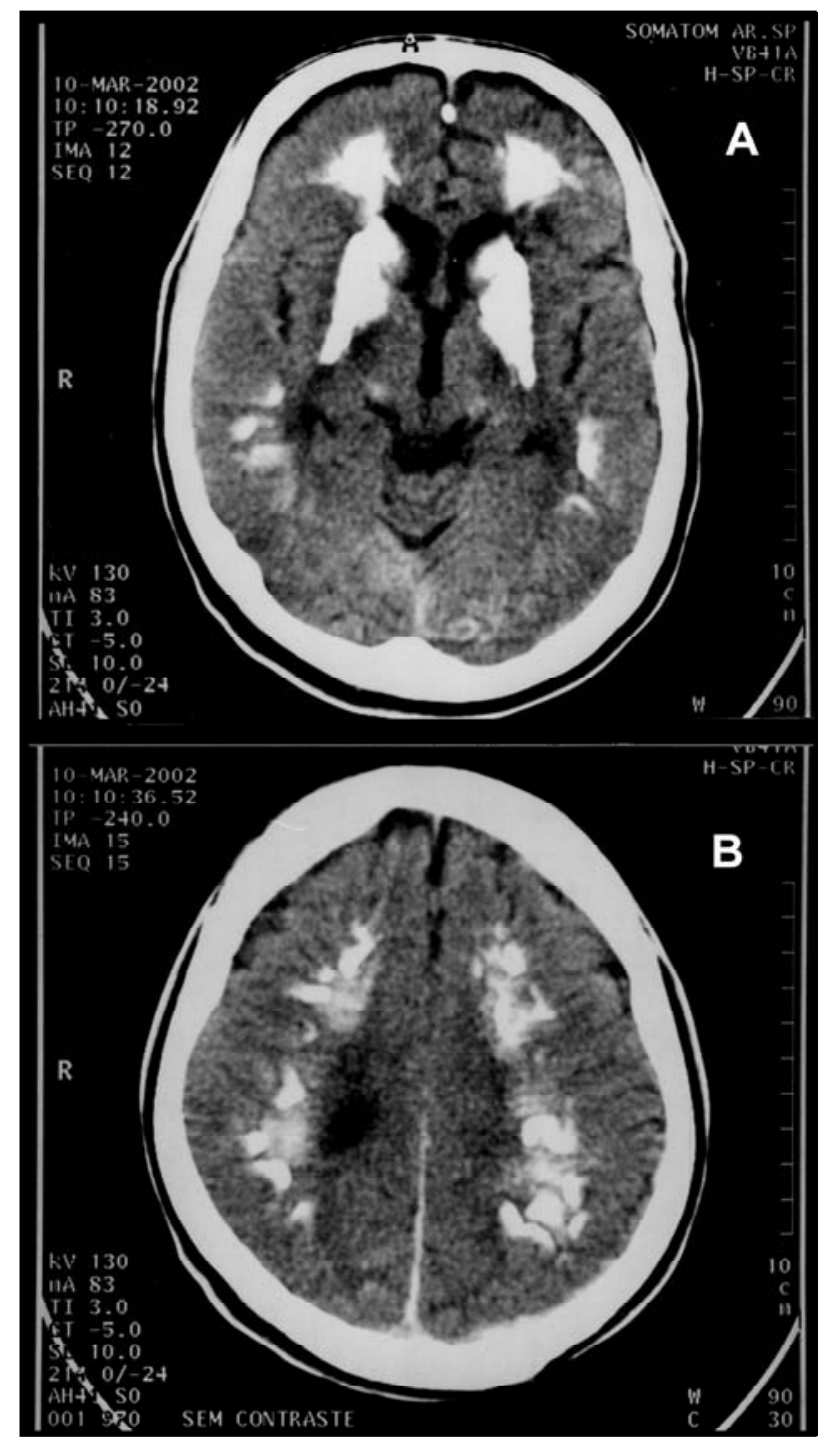

Fig 1. Imagem de tomografia computadorizada de crânio mos trando calcificações difusas, principalmente nos núcleos da ba se e ainda em regiões subcorticais temporais e frontais, frontais p rofundas, nos fórnices menores ( $A$ ) e no centro semioval (B).

siva das alterações do comportamento, exibindo jocosidade, indiferença afetiva e condutas inapropriadas, como urinar no chão da própria casa e comer diretamente nas panelas. Passou a apresentar também tiques fônicos, como pigarrear, e motores, como tocar o nariz com o dedo indicador e a genitália com as mãos. Exibia também comportamento estereotipado de desmontar e remontar fitas cassetes e interruptores de luz elétrica sem objetivo definido, além de andar de bicicleta despropositadamente ("punding"). Mantinha gastos excessivos e rejeitava os cuidados da esposa, tratando-a com impaciência e agressividade. Tentou-se cont rolar a impulsividade e os tiques com haloperidol, mas o paciente mostrou-se muito sensível, desenvolvendo parkinsonismo moderado com apenas $1 \mathrm{mg} / \mathrm{dia}$. Com o mesmo objetivo foi administrada olanzapina até $5 \mathrm{mg} / \mathrm{dia}$, mas não houve alteração significativa no comportamento e o paciente também desenvolveu parkinsonismo. Após dois anos sem 
eventos epilépticos, o fenobarbital foi suspenso. Contudo, exibiu nova crise epiléptica, desenvolvendo, mais uma vez, sintomas cerebelares e parkinsonianos persistentes, pósictais. Esses sintomas melhoraram espontaneamente ao longo de 6 semanas. Optou-se pela introdução do ácido valpróico. Atualmente encontra-se em uso de ácido valpróico $1000 \mathrm{mg} / \mathrm{dia}$ e fluoxetina $20 \mathrm{mg} / \mathrm{dia}$ com controle de crises epilépticas, além de melhora do humor e atenuação da hostilidade dirigida à esposa. Permanecem praticamente estáveis ao longo de seu acompanhamento os tiques, os comportamentos estereotipados e as alterações em sua personalidade. Ressalta-se que não foram utilizadas escalas de avaliação quantitativa de alterações comportamentais e de sintomas extra-piramidais, o que poderia ter contribuído por uma avaliação clínica seriada mais objetiva.

\section{DISCUSSÃO}

Descrevemos o caso de um adulto com DF, de quem foi obtido o consentimento para publicação do caso, cujo quadro se manifestou com transtorno depressivo, mudança da personalidade, comportamentos estereotipados e tiques. O paciente exibiu ainda crises epilépticas, sinais cere bela res e parkinsonianos transitórios.

O quadro clínico da DF é bastante variável, até mesmo quando apresenta ocorrência familiar. Podem ocorrer manifestações extra-piramidais, como parkinsonismo, distonia e tiques, sintomas cerebelares, como ataxia e disartria, epilepsia e síndromes neuropsiquiátricas. As síndromes neuropsiquiátricas mais descritas na literatura são as psicoses esquizofre niformes, os transtornos de humor, os transtornos de personalidade e os sintomas obsessivo-compulsivos. Pode ocorrer também deterioração cognitiva nesses pacientes. Entretanto, a DF pode apresentar-se "assintomática", isto é, sem crises epilépticas, sem sinais motores ou alterações comportamentais ${ }^{4,6}$. Esse fato é importante por mostrar que os circuitos cerebrais podem ter função íntegra apesar do depósito parenquimatoso de substâncias, indicando a possível existência de outros fatores, além da calcificação, gerando as alterações descritas. Ressalta-se que a literatura sobre a DF é p roblemática por incluir, sob a denominação de síndrome de Fahr, outras condições que cursam com calcificação patológica, ou seja, não idiopática, nos núcleos da base.

A DF é paradigmática para os estudos que relacionam os núcleos da base não apenas à motricidade, mas a diferentes aspectos do comportamento humano. Isso porque a DF acomete preferencialmente o globo pálido ${ }^{4}$, embora seja freqüente a calcificação em outras regiões, como no paciente relatado. Outras doenças que afetam os núcleos da base, como a doença de Huntington, a doença de Wilson e a doença de Parkinson, comprometem, respectivamente, o núcleo caudado, o núcleo putâmen e a parte compacta da substância negra. Essas estruturas dos núcleos base, ao constituírem os circuitos frontalsubcorticais, também denominadosfronto-estriatais, facilitariam determinados comportamentos em detrimento de outros comportamentos conflitantes ${ }^{6}$. Não é surpreendente, portanto, que alterações comportamentais reflitam interrupção desses circuitos, sendo comuns nas doenças citadas ${ }^{7,8}$.

Os cinco circuitos fronto-estriatais têm uma organização básica similar. Dois desses circuitos têm funções motoras e três deles possuem funções cognitivas e comportamentais. As conexões do córtex frontal são estabelecidas principalmente com o putâmen, no caso dos circuitos motores, e com o caudado e o núcleo accumbens, no caso dos não-motores. No estriado, o circuito pode assumir uma via direta, conectando o estriado ao segmento interno do globo pálido e à parte reticulada da substância negra (GPi/SNr), ou uma via indireta, ligando o estriado ao segmento externo do globo pálido, em seguida ao núcleo subtalâmico e, finalmente, à GPi/SNr. As duas vias modulam a resposta talâmica ao córtex. Enquanto a direta estimula o tálamo, a segunda o inibe?

A c redita-se que o circuito motor participe da execução automática de seqüências motoras e facilite ou iniba movimentos indesejados, dependendo da ativação das vias direta ou indireta, respectivamente. A geração ou iniciação dos movimentos seria função cortical e cerebelar, enquanto a facilitação dos movimentos pela supressão de padrões conflitantes seria função estriatal ${ }^{7}$. No caso relatado, o paciente apresentou sintomas parkinsonianos transitórios após crises epilépticas e uso de baixas doses de neuroléptico, além de tiques fônicos e motores persistentes, que sugerem comprometimento do circuito fronto-estriatal motor.

O mesmo raciocínio proveniente do circuito motor pode ser extrapolado para os circuitos não-motores fronto-estriatais, nos quais os núcleos da base podem facilitar ou inibir respostas cognitivas, comportamentais ou afetivas. É interessante que, para explicar o fenômeno de seleção de respostas a serem suprimidas ou facilitadas, seria necessário o envolvimento de mecanismos integradores da atividade neuronal, possivelmente mediado por fibras dopaminérgicas originadas na área tegmentar ventral, que constitui a via referida classicamente como mesolímbica ${ }^{7,9}$.

No paciente descrito, pressupõe-se comprometi- 
mento do circuito fronto-estriatal orbital, importante para a tomada de decisões e para a supressão de comportamentos inadequados. Por exemplo, a disfunção da via indireta, inibitória, desse circuito tornaria o indivíduo impulsivo, inadequado e desinibido em relação a recompensas e punições imediatas, ignorando regras sociais, como observado no paciente.

O circuito medial ou límbico, responsável pela experiência emocional e motivação, também pode estar acometido em sua via inibitória neste paciente. Esse tipo de disfunção levaria a um estado de apatia, anedonia e dificuldade de concentrar a atenção, sintomas freqüentes em quadros depressivos e na síndrome negativa da esquizofrenia. $O$ circuito dorsolateral participa do planejamento e da execução de seqüências complexas de ações ou linguagem, sobretudo quando fora da rotina, além de ser fundamental para a memória de trabalho. Ele controla de modo flexível as estratégias de solução de problemas, monitorando o comportamento passo a passo e verificando o próprio desempenho. Alterações nessa região causam disfunções executivas, com perda de iniciativa do ato motor e da espontaneidade, com lentidão e automatismo de respostas, além de comportamento desorganizado resultante da falha no ato de comparar o comportamento produzido com o plano original. Surgem, assim, as perseverações, os estereótipos motores, rigidez e concretude comportamentais, e as marcantes alterações atencionais, de memória operacional e de linguagem ${ }^{7,10}$. O paciente, que apresentava comportamento desorganizado e perseverante, além de comp o rtamentos estereotipados, pode também ter tido esse circuito acometido na sua doença.

Os comportamentos estereotipados denominados "punding" têm sido recentemente descritos na literatura e identificados, até agora, apenas em indivíduos dependentes de anfetaminas ou cocaína e em pacientes com doença de Parkinson (DP) em terapia dopamin é rgica, principalmente naqueles com síndrome de desregulação dopaminérgica ${ }^{5,9,11-13}$.

No primeiro relato de "punding" na DP, Friedman ${ }^{14}$ descreveu o caso de um paciente que mexia repetitivamente em seus papéis, além de cantar "todo o tempo" e contar "piadas sem graça, inapropriadas e sem sentido". Em um estudo específico sob re o fenômeno em pacientes com DP, Evans e colaboradores ${ }^{11}$ descreveram a forma de apresentação dos fenômenos nos pacientes identificados com "pun ding". Um contador não parava de mexer em papéis e gavetas; um carpinteiro fazia reparos desnecessários em sua casa; uma mulher examinava suas jóias incessantemente; uma costureira ficou fascinada por bo- tões; um músico cantava repetitivamente com letras inventadas e outro contador desmontava bicicletas, raramente conseguia remontá-las e fazia passeios ciclísticos sem rumo ou propósito. Homens costumam desmontar equipamentos e organizar, catalogar e analisar suas partes, raramente colocando-as de volta. Mulheres mexem incessantemente em suas bolsas, escovam o cabelo, arrumam-se e lixam as unhas excessivamente. Os pacientes reconhecem que os comportamentos são improdutivos e despropositados. Por outro lado, são considerados apaziguadores e associados à intensa curiosidade, o que leva os pacientes a fazê-los continuamente, ficando agressivos quando interrompidos. Quando envolvidos na atividade ficam absortos, tácitos e não-responsivos, ignorando necessidades básicas como alimentar-se ou eliminações fisiológicas ${ }^{11}$. No paciente que relatamos, o mesmo costumava desmontar e remontar incessantemente interrupto res elétricos e fitas cassetes, dispensando muito tempo nisso, reconhecendo que fazia essas atividades sem propósito e se irritando quando interrompido. Também andava de bicicleta sem rumo e não conseguia conter-se no seu "hábito de contar mentiras e comprar tudo".

O "punding" caracteriza-se por fascinação intensa por tarefas repetitivas que chegam a levar ao isolamento e a conflitos interpessoais. Algumas de suas características o diferem do transtorno obsessivo-compulsivo (TOC). Na definição clássica do TOC, há a int rusão de eventos cognitivos (obsessões) que resultam em comportamentos repetitivos intencionais (compulsões), que visam neutralizar tanto as intrusões cognitivas, quanto a ansiedade associada. Outros fenômenos englobados pelo conceito de espectro obsessivo-compulsivo, como tiques crônicos, síndrome de To u rette, tricotilomania e onicofagia, apesar de não exibirem intrusões cognitivas evidentes, são muitas vezes acompanhados de outros eventos intrusivos, como as intrusões sensitivas ("tiques sensitivos", por exemplo). Diferentemente, os comportamentos descritos como "punding" são dissociados tanto de eventos cognitivos intrusivos, quanto de qualquer outro evento intrusivo.

Além disso, no TOC, as obsessões e compulsões costumam estar inseridas em grupos relacionados à higiene, sexo, violência e ordenação, conforme o contexto sócio-cultural. Assim, o TOC seria o resultado de déficits de processamento implícito de estratégias de procedimentos específicas da espécie presumivelmente armazenadas no estriado ${ }^{13}$. Por sua vez, o "punding" seria o resultado da perda de inibição de hábitos automáticos aprendidos. $O$ conteúdo das ta- 
refas depende da ocupação do indivíduo, seus interesses e passatempos. Os comportamentos do "pun ding" seriam alterações no processamento de estratégias procedurais aprendidas em experiências passadas, ao contrário das estratégias de procedimento específicas da espécie características do TOC. Essa diferença é apontada como essencial na diferenciação dos fenômenos por sugerir o envolvimento de mecanismos neurobiológicos distintos ${ }^{13}$. O fenômeno é subdiagnosticado nos pacientes com DP em reposição dopaminérgica, sendo descritos equivocadamente na literatura como sintomas obsessivo-compulsivos ou mania ${ }^{4,11,13}$.

Em síntese, o paciente que relatamos apresenta diversos sintomas que podem ser relacionados à disfunção dos circuitos fronto-estriatais, como as alterações do comportamento (inadequação e desre speito a normas sociais, jocosidade, agressividade, impulsividade), do humor, tiques e "punding". É importante comentar que essas alterações comportamentais também podem ter sido agravadas pela epilepsia. O melhor controle das crises epilépticas após a int rodução do ácido valpróico coincide, inclusive, com a melhora de alguns dos sintomas comportamentais.

Outro ponto relevante refere-se ao fato de que os sintomas poderiam não estar necessariamente relacionados à topografia das calcificações idiopáticas. Conforme mencionado anteriormente, o depósito intracraniano de cálcio e de outras substâncias pode cursar de forma assintomática. Portanto, "estrutura calcificada" não equivale à "estrutura lesada", ou seja, não há relação inequívoca entre calcificação e sintoma, a despeito de o fluxo sanguíneo estar reduzido em regiões calcificadas do encéfalo ${ }^{4,15}$. A relação estabelecida no caso é pressuposta baseando-se em estudos prévios que correlacionaram os diferentes sinto- mas motores e comportamentais com alterações metabólicas em determinados circuitos fronto-estriatais.

Este trabalho sugere que o estudo da DF, por ser uma doença que acomete essencialmente estruturas subcorticais, juntamente com outras enfermidades que acometem primordialmente os núcleos da base, abre perspectivas para os estudos que relacionam estas estruturas cerebrais a diferentes aspectos do comportamento humano.

\section{REFERÊNCIAS}

1. Geschwind DH, Loginov M, Stern JM. Identification of a locus on ch romosome 14q for idiopathic basal ganglia calcification (Fahr's disease). Am J Hum Genet 1999;65:764-772.

2. Fahr T. Idiopathische Verkalkung der Hirngefässe. Zentralblatt für allgemeine Pathologie und pathologische Anatomie 1930;50:129-133.

3. Faria AV, Pereira IC, Nanni L. Computerized tomography findings in Fahr's syndrome. Arq Neuropsiquiatr 2004;62:789-792.

4. König P. Psychopathological alterations in cases of symmetrical basal ganglia sclerosis. Biol Psychiatry 1989;25:459-468.

5. Law rence AD, Evans AH, Lees AJ. Compulsive use of dopamine replacement therapy in Parkinson's disease: reward systems gone awry? Lancet Neurol 2003;2:595-604.

6. Benke T, Karner E, Seppi K, Delazer M, Marksteiner J, Donnemiller E. Subacute dementia and imaging correlates in a case of Fahr's disease. J Neurol Neurosurg Psychiatry 2004;75:1163-1165.

7. Teixeira-Jr AL, Cardoso F. Neuropsiquiatria dos núcleos da base: uma revisão. J Bras Psiquiatr 2004;53:153-158.

8. Aarsland D, Litvan I, Larsen JP. Neuropsychiatric symptoms of patients with prog ressive supranuclear palsy and Parkinson's disease. J Neuropsychiatry Clin Neurosci 2001;13:42-49.

9. Evans AH, Lees AJ. Dopamine dysregulation syndrome in Parkinson's disease. Curr Opin Neurol 2004;17:393-398.

10. Mattos P, Saboya E, Araújo C. Seqüela comportamental pós- traumatismo craniano. Arq Neuropsiquiatr 2002;60:319-323.

11. Evans AH, Katzenschlager R, Paviour D, et al. Punding in Parkinson's disease: its relation to the dopamine dysregulation syndrome. Mov Disord 2004;19:397-405.

12. Kurlan R. Disabling repetitive behaviors in Parkinson's disease. Mov Disord 2004;19:433-437.

13. Voon V. Repetition, repetition, repetition: compulsive and punding behaviors in Parkinson's disease. Mov Disord 2004;19:367-370.

14. Friedman JH. Punding on levodopa. Biol Psychiatry 1994;36:350-351.

15. Uygur GA, Liu Y, Hellman RS, Tikofsky RS, Collier BD. Evaluation of regional cerebral blood flow in massive intracranial calcifications. J Nucl Med 1995;36:610-611. 